\title{
Polyamines and transglutaminases: biological, clinical, and biotechnological perspectives
}

\author{
Enzo Agostinelli
}

Received: 3 January 2014/Accepted: 27 January 2014/Published online: 20 February 2014

(C) Springer-Verlag Wien 2014

\section{Preface}

The history of polyamines dates back to the fifteenth century when spermine was discovered by Antoni van Leeuwenhoek [born in Delft, Holland (1632-1723)], and yet it took many years before serious attention was given to understanding the role of spermine or other polyamines in the biology of living cells. It is now clear that regulation of polyamine homeostasis is complex and has excited polyamine researchers who have continued to focus on this productive area of research. Therefore, enough new research findings have prompted to organize conferences and congresses worldwide to disseminate the new knowledge generated. New research in the areas of biosynthesis and action of the ubiquitous polyamines, with particular focus on the biology of normal and cancerous eukaryotic cells, and plants, was the driving force to hold a 6-day International Congress on "Polyamines Biological and Clinical Perspectives" in Turkey at Istanbul Kultur University, in the beautiful city of Istanbul, from 2 September to 7 September, 2012. This conference was organized by Professor Narcin Palavan Unsal (Istanbul Kultur University) and Professor Enzo Agostinelli (SAPIENZA University of Rome, Rome, Italy).

Istanbul is a largest marvelous city in Turkey. Located on both sides of the Bosporus, Istanbul bridges Asia and

\footnotetext{
E. Agostinelli $(\bowtie)$

Istituto Pasteur-Fondazione Cenci Bolognetti, Department of Biochemical Sciences, SAPIENZA University of Rome,

Piazzale Aldo Moro 5, 00185 Rome, Italy

e-mail: enzo.agostinelli@uniroma1.it

E. Agostinelli

Institute Biology and Molecular Pathology, CNR, Piazzale Aldo

Moro 5, 00185 Rome, Italy
}

Europe. The ancient name of Istanbul was Bisantium, a city founded by Greeks in 659 B.C. on the banks of the Bosporus. Bisantium was renamed Constantinopolis in honor of the Roman emperor Constantine I, becoming a center of Greek culture and Christianity. Throughout its long history, Istanbul (the old Constantinopolis) was the capital of three important empires: Roman, Byzantine, and Ottoman. Today, Istanbul as one of the largest cities in the world is also one of the European capitals of culture while its historic areas are part of the UNESCO list of World Cultural Heritage.

This Special Issue of amino acids brings together 28 peer-reviewed manuscripts that provide the essence of the lectures and posters presented at the above-mentioned conference on the biological and physiological roles of Polyamines and Transglutaminases (TGases), as well as a few manuscripts authored by internationally recognized experts who could not attend the meeting. All the articles deal with high-class research data obtained until the mid2013. ${ }^{1}$ This issue also provides short overviews of some important concepts and notions in the subject matter, which represent important tools for those new investigators who may embark on investigating this intriguing field. The papers project the role of polyamines in cell growth and differentiation, cell cycle regulation, gene expression and signal transduction in animals, plants and microorganisms as well as in several pathophysiological processes including carcinogenesis and other diseases. Recently, the significance of polyamines in cell proliferation and carcinogenesis has stimulated further research interest in their biochemical and metabolic regulation at genomic level (Zhu et al. 2012; Cervelli et al. 2013; Murray-Stewart

\footnotetext{
${ }_{1}$ All manuscripts in this special issue were subjected to external peer reviewing according to the policy of this journal.
} 
et al. 2013; Pasini et al. 2013). Inhibition of polyamine biosynthesis as an antitumor strategy has been demonstrated to be generally ineffective in clinical trials, but there is some potential in human diseases like cancer (Raj et al. 2013) and parasitic diseases (Bacchi et al. 2009; Preeti et al. 2013; von Koschitzky and Kaiser 2013). It is clear that questions on polyamine action should be unraveled at molecular level to clarify the significance of polyamines in cell homeostasis related to various biological conditions. For this reason, this issue is not restricted to human diseases alone, but also includes a number of studies carried out on plant growth and regulation as well as evolution patterns in microorganisms.

\section{Editorial}

This is the fourth special issue of this journal focused on biogenic amines, the polyamines. As mentioned above, it is an editorial initiative for publishing the recent findings on biological roles of polyamines and transglutaminases (TGases), a majority of which were presented at the international meeting held in Istanbul (Turkey) in 2012. The contents of this current amino acids special issue reflect the current knowledge and future perspectives of the physiological, biochemical, and therapeutic actions of both polyamines and transglutaminases.

Cellular polyamine concentrations are highly regulated. Intracellular accumulation of polyamines, due either to high extracellular levels or deregulation of the systems that control polyamine homeostasis, can induce programmed cell death (or apoptosis) in various cell types (Tobias and Kahana 1995; Pignatti et al. 2004; Seiler and Raul 2005). The natural polyamines spermidine and spermine are substrates of several enzymes that generate cytotoxic metabolites: monoamine oxidase (MAO), polyamine oxidase (PAO), spermine oxidase (SMO), and copper amine oxidases (CuAOs) (Arancia et al. 2004; Agostinelli et al. 2004). Amine oxidases (AOs) are important because they regulate the levels of these polycations. PAO is involved in the homeostatic regulation of polyamine pools, while other oxidases are important for the terminal catabolism of polyamines, i.e. they catalyze the formation of metabolites that are excreted via the kidneys (Seiler 1992). Products of polyamine oxidation are toxic to cells. Mono-, di- and poly-amines, as well as several $\mathrm{N}$-acyl amines, are oxidatively deaminated by AOs in a reaction consuming $\mathrm{O}_{2}$ and $\mathrm{H}_{2} \mathrm{O}$. In fact, experiments were performed to induce cytotoxicity in several human tumor cell lines in vitro using purified bovine serum amino oxidase (BSAO), a CuAOs, in the presence of exogenous spermine or endogenous polyamines (Calcabrini et al. 2002; Agostinelli et al. 2009) or after injection of the enzyme into the tumor in vivo
(Averill-Bates et al. 2005). Amine oxidases preferentially use polyamines (spermine and spermidine) as substrate to generate the reactive oxygen species (ROS), $\mathrm{H}_{2} \mathrm{O}_{2}$, and aldehyde(s) (Agostinelli and Seiler 2006a). These toxic products are able to induce stress-activated signal transduction pathways, leading to cell death by necrosis or apoptosis (Lindsay and Wallace 1999; Seiler and Raul 2005; Agostinelli et al. 2006b). These findings have a potential clinical application in cancer therapy (Arancia et al. 2004; Agostinelli et al. 2004, 2010; Averill-Bates et al. 2008; Vijayanathan et al. 2013; Amendola et al. 2013). In light of this, a novel kind of super paramagnetic maghemite nanoparticles (SAMNs, surface active maghemite nanoparticles) were modified with BSAO, using rhodamine-isothiocyanate adduct as fluorescent spacer arm. A fluorescent and magnetically drivable adduct comprising copper containing bovine amine oxidase immobilized on the surface of specifically functionalized magnetic nanoparticles was developed (SAMN@RITC-BSAO). The multifunctional nanomaterial was characterized by transmission electron microscopy, infrared spectroscopy, mass spectrometry, and activity measurements. These analyses suggested that SAMN@RITC-BSAO complex with a specific activity of $0.81 \mathrm{IU} \mathrm{g}^{-1}$ could be used, in the presence of polyamines, as fluorescent magnetically drivable $\mathrm{H}_{2} \mathrm{O}_{2}$ and aldehyde(s) (acrolein) producing system. Future applications in selectively destroying tumor cells have been suggested (Sinigaglia et al. 2012).

Post-translational modifications of proteins via covalent binding of polyamines with special emphasis on TGases and hypusine biosynthesis are also highlighted in this issue. TGases are ubiquitous enzymes with multifaceted functions. They were identified when a liver enzyme was found to incorporate amines into proteins (Sarkar et al. 1957). An acyl-enzyme thioester intermediate is formed between an active site cysteine and polypeptide-bound glutamine, and then the thioester intermediate reacts with a suitable nucleophile is the mechanisms involved (Folk and Cole 1966). Current research on TGases includes both basic and applied aspects, involving the fields of medicine (e.g., development of diseases such as cancer and Alzheimer), plant science (e.g., regulation of processes such as photosynthesis and fertilization), and food and material sciences.

Tissue transglutaminase (TG2) is the most ubiquitous member of the mammalian transglutaminase (TG) family. This multifunctional calcium-dependent enzyme catalyzes the post-translational protein modification of proteins leading to the formation of intra- or inter-molecular epsilon (gamma-glutamyl) lysine bonds (cross-links), or polyamine incorporation into proteins (Folk et al. 1980; Griffin et al. 2002). In addition, TG2 can bind and hydrolyse GTP and displays protein disulphide isomerase activity as well as a 
protein kinase activity (Park et al. 2010). Given the pleiotropic functions of this protein, TG2 has been implicated in a variety of events including the suppression of cell proliferation, cell differentiation, signal transduction, apoptosis, and wound healing (Fesus and Piacentini 2002; Telci and Griffin 2006). The role played by TG2 in the cell response to redox state imbalance has been reviewed by Caccamo et al. (2012). The study was performed under both physiological and pathological conditions, such as neurodegenerative disorders, inflammation, and autoimmune diseases, in which oxidative stress plays a pathogenic role, accelerating the progression of the disease.

The first part of the issue is entirely devoted to polyamine catabolism and its role in animals and plants. The physiological functions of these amines have yet to be elucidated completely at the molecular level. Nonetheless, various effects of polyamines in biology are well known and, in several instances, the mechanisms underlying these effects have been clarified. Since the polyamine biosynthetic pathway is very active during the growth of various cancer cells, it was found that polyamines are often present at high concentrations in rapidly dividing tumor cells and growing tissues. Therefore, special attention has been given to their contribution to carcinogenesis and development of new approaches to the therapy of cancer and of other diseases. Success in therapy has been obtained in some endemic parasitic protozoan effects, as in trypanosomiasis by difluoromethylornithine (DFMO).

Combination therapy targeting polyamine metabolism could represent a promising strategy to fight hyper-proliferative disease and use delivery systems to obtain differential response between healthy cells versus cancer cells. DNA damage provoked by enzymatic ROS overproduction may act as an additive or adaptive response upon radiation while combination of hyperthermia with lysosomotropic compounds may improve the cytocidal effect of polyamine-derived oxidative metabolites. BSAO and spermine association in multidrug resistant cells, sensitized to spermine metabolites by an increased mitochondrial activity, and mammals spermine oxidase are differentially activated in tissues and cancer cells (Amendola et al. 2013). Another approach to inhibit cancer cell growth is the use of polyamines for DNA transport for gene therapy (Vijayanathan et al. (2013). Natural polyamines interact with nucleic acids by virtue of their primary, secondary and tertiary amino groups that are positively charged under physiological $\mathrm{pH}$ conditions (Thomas and Thomas 2001). A consequence of the interaction of spermidine and spermine with DNA is the condensation of DNA to nanoparticles, of approximately $100 \mathrm{~nm}$ diameter, to facilitate the cellular uptake of nucleic acids (Vijayanathan et al. 2002). Future studies are, therefore, aimed to test if a prerequisite for DNA transport to cells is the condensation of
DNA to nanoparticles (Thomas et al. 1999). That polyamine catabolism is critical for the maintenance of polyamine homeostasis in normal tissue is also reviewed (Battaglia et al. (2013). It should be noted, however, that there are specific disease states where the dysregulation of polyamine catabolism is associated with the etiology and progression of cancer. The article by Cervelli et al. (2013) is an overview on genetically engineered rodents that were generated and utilized for studies to assign specific cellular roles for polyamines and their metabolic enzymes in cellular inflammation, cancer development, and brain disorders. The studies indicate that alteration of polyamine metabolism in transgenic and gene-disrupted animals has created a number of animal models relevant to human diseases. For the first time, a transgenic mouse line conditionally overexpressing spermine oxidase in cerebral cortex, which represents a new genetic model for studying brain pathologies and testing new pharmacological therapies, is discussed. The review article by Chaturvedi et al. (2013) summarizes the importance of the utilization of Larginine in the host response to the gastric pathogen $\mathrm{Hel}$ icobacter pylori. The authors show that $H$. pylori is a gastric pathogen that causes gastric inflammation, peptic ulcers, and gastric cancer. Their aim was to determine if modulation of spermine levels by spermine oxidase (SMO) plays a role in host innate immune response. Using SMO knockdown and overexpression strategies, the authors show that SMO can effectively enhance nitric oxide (NO) production in macrophages, by depleting spermine sufficiently to facilitate cellular uptake of L-arginine that is required as the substrate for inducible NO synthase (iNOS) and for iNOS protein expression. SMO-dependent NO release results in killing of $H$. pylori, leading to a novel link between spermine metabolism and host defense.

Active polyamine biosynthetic pathway during the growth of various cancer cells and high polyamine levels associated with rapidly dividing tumor cells and growing tissues seem to be a result of increased levels ornithine decarboxylase (ODC), the rate-limiting enzyme, causing enhanced putrescine synthesis from ornithine as well as by increased uptake of polyamines (Marton and Pegg 1995). Some articles of the issue are devoted to this enzyme. First evidence has been obtained that elevated levels of polyamines alone can stimulate the recruitment of epidermal bulge stem cells Hayes et al. (2013). This is a significant finding with regard to the stem cell origin of skin cancer. Because elevated ODC activity in keratinocytes also stimulates ROS generation via induction of polyamine catabolic oxidases, the authors investigated the contribution of these toxic polyamine catabolic metabolites to polyamine-dependent tumor development originating from dormant bulge stem cells that possess carcinogen-initiated mutations. These data offer further insight into the role of 
polyamines in promoting skin tumorigenesis. Apoptotic cell death induced by epibrassinolide (EBR), a biologically active compound of the brassinosteroids, related to polyamine biosynthetic and catabolic pathways in LNCaP $(\mathrm{AR}+), \mathrm{DU} 145(\mathrm{AR}-)$ prostate cancer cell lines and PNT1,a normal prostate epithelial cell line, has been investigated (Obakan et al. (2013). Induction of apoptotic cell death was observed in prostate cancer cell lines after EBR treatment. In addition, EBR induced the decrease of intracellular polyamine levels accompanied by a significant ODC downregulation in each prostate cancer cells and also modulated ODC antizyme (OAZ) and antizyme inhibitor (AZI) expression levels only in LNCaP cells. Catabolic enzymes spermidine/spermine acetyltransferase (SSAT) and PAO expression levels were up-regulated in both cell lines; however, the specific SSAT and PAO siRNA treatments prevented the EBR-induced apoptosis only in LNCaP $(\mathrm{AR}+)$ cells. In a similar way, the co-treatment of $\mathrm{N}^{1}, \mathrm{~N}^{4}$-bis(2,3-butadienyl)-1,4-butanediamine (MDL) 72527, the specific PAO and SMO inhibitor, with EBR during $24 \mathrm{~h}$, reduced the formation of cleaved fragments of PARP in LNCaP (AR+) cells. That amino acids like asparagine (ASN) and glutamine (GLN) may act as hormones is featured in a review by Ray and Johnson (2013). It is known for years that amino acids like ASN and GLN are essential for the growth factors to induce ODC activity (Chen and Canellakis 1977; Ray et al. 1999). The finding that they act by inhibiting AZ synthesis involving TORC2 provides a mechanism for their effects. Recent evidence suggesting that AZ directly stimulates apoptosis and inhibts proliferation, so that its synthesis can be regulated through the mTOR pathway. These amino acids may have direct effects on tissue growth independent of their nutrient value (Fong et al. 2003; Ray et al. 2012).

Antizyme inhibitors (AZI/AZIN) are key regulators of the polyamine pathway. AZIs interfere with antizyme functions, leading to increased polyamine biosynthesis, increased cell proliferation, as well as enhanced cell transformation and tumorigenesis (Mangold 2006; KerenPaz et al. 2006). Murakami et al. (2013) provide evidence that multiple forms of mouse antizyme inhibitor $1 \mathrm{mRNA}$ are differentially regulated by polyamines. The authors found various Azin1 transcripts formed by alternative splicing or alternative transcription initiation in mice. Polyamines regulate Azin1 expression at the transcription initiation and selection of splicing acceptor sites for the exon 7 , both of which may affect the level of mRNA encoding the active Azin1 protein.

Previously, polyamine analogues were designed, synthesized, and tested as anticancer agents (Casero and Woster 2009) as well as in neurodegenerative processes (Minarini et al. 2010), antiparasitic compounds, neurotransmitter receptor neuroprotectants, and multitarget- directed ligands for multifactorial diseases. Murray-Stewart et al. (2013) report the effects of an antitumor polyamine analogue as an inhibitor of LSD1 in acute myeloid leukemia (AML). The results indicate a re-expression of the aberrantly silenced $e$-cadherin tumor suppressor gene as a direct result of LSD1 inhibition and the associated changes in local chromatin structure. The effects on the LSD1 inhibitor on cellular proliferation and polyamine metabolism were also reported. The authors concluded that the use of polyamine analogues as inhibitors of LSD1 is a promising approach to the treatment of AML. Dysregulation of epigenetic control of gene expression is a common feature in the initiation and progression of cancer. DNA methylation and covalent modification of histones collaborate to function as epigenetic regulators of gene expression. Pasini et al. (2013) offer an overview of the literature regarding polyamine influence on the modulation of chromatin arrangement participating into the transcriptional regulation of gene expression. Polyamine derivatives acting as epigenetic modulators are also presented since these novel polyamine-based pharmacologically active molecules are promising tools in anticancer therapy.

Polyamines are readily interconverted which complicates studies on the functions of the individual polyamines. Thus, non-metabolizable analogues, like carbon-methylated polyamine analogues, are needed to circumvent that problem. Keinänen et al. (2013) have reviewed methylated putrescine, spermidine, and spermine analogues in which at least one hydrogen atom attached to polyamine carbon backbone has been replaced by a methyl group. The authors observed that methylated polyamine analogues allow the regulation of both metabolic and catabolic fates of the parent molecule in vitro and in vivo. Substituting the natural polyamines with methylated analogue(s) offers means to study either the functions of an individual polyamine or the effects of altered polyamine metabolism on cell physiology. In this regard, Castro-Oropeza et al. (2013) report an interesting application of polyamine analogues. They observed that histamine plays important roles in the development of many emergent and rare diseases. In mammals, histamine is formed by decarboxylation of L-histidine, which is catalyzed by pyridoxal-5'-phosphate (PLP) dependent histidine decarboxylase (HDC). The reduced availability and stability of the protein have delayed the characterization of its structure-function relationships. The authors in their previous rHDC knowledge, derived from both in silico and experimental approaches, indicated that an effective competitive inhibitor of HDC should be capable to form an "external aldimine-like structure" and have an imidazole group, or its proper mimetic, which provides additional affinity of PLP-inhibitor adduct to HDC active center. This was confirmed by using aminooxy analogue of histamine, having IC50 of 
$2 \times 10^{-7} \mathrm{M}$ and being capable to form PLP-oxime in the enzyme active center.

Understanding the role of highly conserved proteins across evolution, like those involved in protein synthesis, is essential not only to establish the basis of the mechanisms involved in the regulation of gene expression, but also to help modulate such processes during disease conditions. Eukaryotic initiation factor 5A (eIF-5A) is one of these proteins and constitutes an interesting drug target. eIF-5A is a regulatory protein that upon posttranslational modification controls translation and subsequent protein biosynthesis. The mechanisms which lead to enhanced proliferation are unknown. Modification of eIF-5A involves two catalytic steps by deoxyhypusine synthase (DHS) and deoxhypusine hydroxylase (DOHH). Hitherto, a rapid and robust assay to differentiate between the modified and unmodified form did not exist. Nishiki et al. (2013) achieved an analytical breakthrough with a polyclonal antibody (IU-88) that specifically recognizes only the hypusinated modified form of eIF-5A. Novel findings emphasize that eIF-5A can be downregulated by RNAi (Schwentke et al. 2012) but deletion mutants are lethal, emphasizing its role in posttranslational modification (Nishimura et al. 2012). Modification of eIF-5A by hypusine is linked to different pathways and is preferentially regulated under stress conditions (inflammation, hypoxia, abiotic stress in plants). This results in translational control of specific mRNAs in diabetes and infectious diseases (Hauber et al. 2005; Chawla et al. 2012; Atemnkeng et al. 2013; Bresciani et al. 2013), pinpointing its important role in translational medicine. The study by Tersey et al. (2013) tested the hypothesis that polyamines may contribute to inflammation and autoimmunity in type 1 diabetes. The authors showed that treatment of type 1 diabetes-prone mice with the ornithine decarboxylase inhibitor DFMO during a discrete period of time, when autoimmunity and inflammation are thought to accelerate, resulted in a reduction in later diabetes incidence. They observed that treated mice display improvements in insulin secretion, reductions in beta cell inflammation, and reductions in pathogenic Th17 cell numbers. These studies point to a role for polyamines via either hypusine modification of eIF-5A or in promoting general mRNA translation, in the pathogenesis of inflammation. Moreover, the studies suggest that approaches that reduce polyamine accumulation or eIF-5A hypusination may be novel adjunctive therapies in type 1 diabetes.

A first approach to discover new antimalarials has been recently performed with the "antimalarial toolbox" from GSK (von Koschitzky and Kaiser 2013). A selection of these compounds with a potential in iron chelating capacity will be applied to inhibit DOHH, a metalloprotein which consists of ferrous iron and catalyzes the second step of the posttranslational modification of eukaryotic initiation factor 5A (EIF-5A) by hypusine. For a High Throughput
Screening (HPTS), a proteomic platform for the detection of hypusine metabolites was established. The identified hits can be tracked to test their efficacy in novel "in vitro assays" against hypnozoites from $P$. vivax and gametocytes $P$. vivax and $P$. falciparum. Rossi et al. (2013) used the yeast model and genetic analysis to confirm a role for eIF$5 \mathrm{~A}$ in translation elongation. Using protein reporters and genetic interactions, the authors demonstrated that a yeast mutant of eIF-5A does not affect the posttranslational pathway of protein translocation into the endoplasmic reticulum (ER), but does affect the co-translational pathway. They also found that this effect is not a result of general block of translation elongation and that eIF-5A function is necessary for efficient binding of signal recognition particle (SRP) to ribosomes.

In the recent years, our understanding of the importance of membrane transporters (MTs) in the disposition of and response to drugs has increased significantly. MTs are proteins that regulate the transport of endogenous molecules and xenobiotics across the cell membrane. In mammals, two super-families have been identified: ATPbinding cassette (ABC) and solute carrier (SLC) transporters. There is evidence that MTs might mediate polyamines (PA) transport. In mammalian cells, three major PA are synthesized, putrescine (Put), spermidine (Spd) and spermine (Spm); while, the decarboxylated arginine (agmatine/Agm) is not produced by mammals but is synthesized by plants and bacteria. In addition, research in the PA field suggests that PA are transported into cells via a specific transporter, the Polyamine Transport System(s) (PTS). Although the PTS has not been fully defined, there is evidence that some of the known MTs might be involved in PA transport.

Abdulhussein and Wallace (2013) have reviewed eight SLC transporters and their potential to mediate PA transport in human cells. These transporters are SLC22A1, SLC22A2, SLC22A3, SLC47A1, SLC7A1, SLC3A2, SLC12A8A, and SLC22A16. Preliminary experimental data by the authors suggest that SLC22A1 might be involved in the PA uptake; in addition, one member of $\mathrm{ABC}$ superfamily (MDR1 protein) might also mediate the efflux of polyamine like molecules. Terui et al. (2013) present an interesting study on the properties of putrescine uptake. Physiological significance of two putrescine transport systems, PotFGHI and PuuP, in Escherichia coli was studied. The results have indicated that Pot FGHI is necessary to stimulate rapid cell growth in the presence of glucose as an energy source. Instead, in the absence of glucose in medium, PuuP was necessary when putrescine was used as an energy source.

The potential role of polyamines in regulating cell proliferation and death have induced scientists to investigate the properties of these polycations in mitochondria, 
multifunctional organelles participating in a range of cellular processes such as energy production, proliferation, senescence, and death (Goldenthal and Marin-Garcia 2004). Mitochondria apparently lack a polyamine biosynthetic pathway; nevertheless, substantial quantities of spermine and spermidine have been detected in the mitochondrial matrix and a specific mitochondrial polyamine transporter has been described (Toninello et al. 2004). Grancara et al. (2013) report that the polyamine spermine is transported into the mitochondrial matrix by an electrophoretic mechanism whose driving force is the electrical membrane potential $\Delta \Psi$. The transport system is a specific uniporter constituted by a protein channel exhibiting two asymmetric energy barriers. Although spermine transport is electrophoretic in origin, its accumulation does not reach the Nernst equilibrium as the polyamine also goes out from mitochondria. Spermine efflux may be induced by different agents able to completely, or partially, reduce the $\Delta \Psi$ value. However, this efflux may also take place in normal conditions when the electrophoretic accumulation of the polyamine induces a sufficient drop in $\Delta \Psi$ able to trigger the efflux pathway. The release of spermine is most probably electroneutral in origin and can take place in exchange with protons or in symport with phosphate anion. The presence of the efflux pathway is consistent with a continuous cycling of spermine across the mitochondrial membrane. This event may assume important effects at the level of mitochondrial permeability transition modulation and consequently on the triggering of intrinsic apoptosis. Weiger and Hermann (2013) review the physiology of cell proliferation, polyamines and potassium channels and their interactions. They describe the importance of polyamines as well as of potassium channels for cell proliferation and cell cycling and the modulation of potassium channels by polyamines. The authors speculate that there might be a feedback loop between these two players where membrane potential is regulated by polyamines and also a feedback from membrane potential to polyamines.

As reported above, spermidine/spermine $N^{l}$-acetyltransferase (SSAT) is a key catabolic enzyme of polyamine metabolism. Pirnes-Karhu et al. (2013) provided data on mice overexpressing SSAT that showed disturbed hematopoietic lineage commitment. SSAT overexpression and the concomitantly accelerated polyamine metabolism in hematopoietic cells and bone marrow microenvironment lead to the development of a mouse myeloproliferative disease in SSAT mice. Liu et al. (2013) investigated the mechanism for the linkage between polyamine metabolism and fat homeostasis that was recently discovered to operate in mice. A system biology approach was used to support their hypothesis that the polyamine flux generated by an increase in SSAT activity in the adipose tissue consumes the SSAT substrate, acetyl-CoA and results in a lean phenotype. Conversely, deletion of SSAT in adipose tissue diverts the acetyl-CoA from polyamine catabolism toward fat synthesis and results in the obese phenotype. Moreover, their data show that the cells can sense the levels of acetylCoA to regulate the enzymes in the glucose metabolism to maintain an adequate concentration of this key metabolite. Borzì et al. (2013) mini-review focuses on polyamine delivery as a tool to modulate stem cell differentiation in skeletal tissue engineering. They examine growing literature suggesting a role for polyamines in bone development and osteogenesis, by enhancing differentiation of both stem cells and differentiated chondrocytes. Therefore, it is tempting to suggest their use as a tool for improving regenerative medicine strategies in orthopedics.

As in animals, spermidine and spermine are synthesized in plants by aminopropyltransferases (APTs) that transfer aminopropyl moieties, derived from decarboxylated $S$-adenosylmethionine, to putrescine and spermidine to produce spermidine and spermine, respectively. In plants, polyamines play pivotal roles in a wide range of growth, developmental, and defense processes (Kusano et al. 2008; Handa and Mattoo 2010). Indeed, changes in polyamine levels occur during certain plant developmental transitions or exposure to stress conditions. In addition, exogenous applications of polyamines have frequently been shown to affect plant growth and response to various biotic and abiotic stresses. Lasanajak et al. (2013) provide a novel contribution using flux studies for better understanding the physiological roles of polyamines and their metabolic pathways vis a vis other hormones in plant growth, development, and senescence. $S$-adenosylmethionine (SAM), a major substrate in 1-C metabolism, is a common precursor in the biosynthetic pathways of polyamines (PAs) and ethylene $\left(\mathrm{C}_{2} \mathrm{H}_{4}\right)$, two important plant growth regulators that share SAM for their biosynthesis. However, the flux of various substrates into these pathways in plants was not yet characterized. The authors used radiolabeled ${ }^{14} \mathrm{C}$-Arg, ${ }^{14} \mathrm{C}-$ Orn, L-[U- $\left.{ }^{14} \mathrm{C}\right] \mathrm{Met},{ }^{14} \mathrm{C}-\mathrm{SAM}$ and ${ }^{14} \mathrm{C}$-Put to quantify flux through these pathways in tomato fruit and evaluate the effect of perturbing these pathways in transgenic tomatoes expressing a yeast SAM decarboxylase (ySAMDC) in a fruit-specific manner. Taken together, the results show that SAM decarboxylase is a rate-limiting enzyme and the decline in Spd and Spm levels during ripening/aging of fruit can be reversed through expression of the heterologous SAM decarboxylase without significantly altering $\mathrm{C}_{2} \mathrm{H}_{4}$ biosynthesis. These studies provide an insight into the nature of metabolic regulation and its homeostasis involving these two, generally antagonistic, plant growth regulators. Shao et al. (2013) have compared catabolic turnover rates of spermidine and spermine in control and high putrescine-producing transgenic poplar cells and Arabidopsis plants using pulse-chase experiments 
with labeled polyamines. The half-life of spermidine was found to be 23-32 h in poplar and 52-56 h in Arabidopsis, and that of spermine around $24 \mathrm{~h}$ in Arabidopsis and $36-48 \mathrm{~h}$ in poplar. The catabolism of spermidine and spermine was four to sixfold slower than that of putrescine. A 10 to 50-fold increase in putrescine had little effect on the turnover of spermidine and spermine.

The second part of this issue is dedicated to novel aspects of TGases. The aberrant induction of TG2 activity contributes to various pathologies, including neurodegenerative diseases, atherosclerosis, autoimmune diseases, and fibrosis (Kim et al. 2002; Szondy et al. 2011). Also, studies on plant TGases and their application in material science and as potential biotechnological tool kits are included.

The interest in TGases has been growing hugely during the past years, as witnessed by the number of reviews and by appearance of Journal special issues dedicated to the enzymes, as is the case for Amino Acids ( $\mathrm{Vol} \mathrm{44}$, issue 1, edited by Beninati et al. (2013)). This blossoming follows directly the discoveries that the enzyme can be involved in autoimmune and in inflammatory diseases (celiac disease, tissue inflammation, and fibrosis are significant examples), that it is relevant for neoplastic growth, and that it can be employed also as target for therapeutic intervention, eventually to modify potential allergens and drugs affecting their bioavailability and clearance mechanisms.

What is now astonishing is the concept that TGases (and particularly the type 2 enzyme that is a multifunctional protein) can perform several and frequently contrasting roles, among them, e.g., promotion of either cell survival or cell death, promotion or suppression of tumor spreading, acceleration or protection from inflammation processes. One attractive possibility is that these pleomorphic actions of TGases are linked to selective triggering of alternative activities (Bergamini et al. 2011; Gundemir et al. 2012) such as transamidation, G-protein signaling, scaffolding, or interaction with other proteins because of alternative splicing or location to different environments including intra- or extracellular space, to which it can gain access through still undefined pathways. Three communications focus these aspects. Currò et al. (2013) have demonstrated that TG2 expression increased in the inflammatory cell response and TG-mediated post-translational modification is involved in signal transduction associated with cell activation. They have characterized the interactions between TG2 and PLA 2 in LPS-stimulated THP-1 cells, a model of monocyte/macrophage differentiation. The results showed that TG2 increases a sustained activation of PLA $\mathrm{P}_{2}$ activity. The functional interaction between these enzymes should be a relevant mechanism in the regulation of inflammatory response. TGase catalyzes acyl transfer reactions between the gamma-carboxamide groups of protein-bound glutamine residues, which serve as acyl donors, and primary amines, resulting in the formation of new gamma-amides of glutamic acid and ammonia. And the review by Mariniello et al. (2013) addresses the use of TGase to covalently modify proteins of industrial interest, via the attachment of aminated polymers, such as polysaccharides, cyclodextrins, and polyethilenglicole.

Besides representing widely distributed enzymes with pleiotropic functions in animals, TGases have important roles in plant science. TGases have been detected in algae and angiosperms; the main TGase reaction studied in plants has been the post-translational modification of proteins by the conjugation with polyamines. In "higher" plants, extracellular TG appears to be involved in cell wallstrengthening, i.e., in flower petals, where TG could be responsible for the corolla strengthening. In pollen cell walls, an extracellular TG is necessary for tube apical growth (possibly for the building of the new cell wall). Even though TG seems to be secreted through vesicles by an actin filament-based membrane trafficking, the site of release and alternative mechanism of secretion remain to be clarified. Up to date scarce information is available on the role of extracellular TGase particularly in plant cell. Interestingly, Del Duca et al. (2013) mainly focus on the possible role(s) of this extracellular enzyme.

In conclusion, the interest in polyamine and TGase function has continued with a new crop of researchers, who have replaced several authoritative scientists in these fields some of whom have sadly passed way. As is the nature of the things and how progress is made, the foundations laid by the previous scientists will remain a cornerstone for the new generation of scientists to reveal still new and unexplored facets of the function of polyamines and TGases.

Acknowledgments The editor wishes to thank all the participants and institutions for their kind contribution to the International Congress on "Polyamines Biological and Clinical Perspectives" organization. Thanks are due to the Rector, Prof. Dursun Kocer, who allowed the meeting to take place at Istanbul Kultur University, Turkey, and to the Rector, Prof. Luigi Frati, SAPIENZA University of Rome for his kind support. The Editor also thanks Professor Narcin Palavan Unsal, chair of the meeting, and her collaborators Drs. E. Damla Arisan, Ajda Coker-Gurkan, Pinar Obakan, and Pelin Ozfiliz, Department of Molecular Biology and Genetics, Istanbul Kultur University, for their great effort for the success of the meeting. I am particularly grateful to all the Authors, mainly to the young scientists, for their scientific support in making this a reality. The Editor also expresses his gratitude to Professor Gert Lubec, Editor-inChief, for the opportunity to publish this special issue in Amino Acids and to Drs. Autar Mattoo, Carlo Bergamini, and Annette Kaiser for their helpful suggestions and acknowledges the editorial help of his collaborator Dr. Giampiero Tempera, who assisted in reading the revised manuscripts that comprise this large volume. The grant support from Italian MIUR (Ministero dell'Istruzione, dell'Università e della Ricerca), Istituto Superiore di Sanità "Project Italy-USA", Istituto Pasteur-Fondazione Cenci Bolognetti and MIUR-PRIN (Cofin) is gratefully acknowledged. Thanks are also due to Fondazione 'Enrico ed Enrica Sovena' for the scholarship given to Giampiero Tempera for supporting his post.doc. 
Conflict of interest The author declares that he does not have conflict of interest.

\section{Appendix: Contributors}

Abdulhussein A.A.

Agostinelli E.

Alhonen L.

Amendola R.

Andrews B. J.

Angelucci E.

Arısan E.D.

Asim M.

Barrero C.

Barry D.P.

Battaglia V.

Bellato H.M.

Bhatnagar P.

Boldrin P.

Bolkent S.

Borzì R.M.

Bragadin M.

Caccamo D.

Cai G.

Calcabrini A.

Caldarera C.M.

Casero R.A.

Castro-Oropeza R.

Cervelli M.

Cetrullo S.

Chaturvedi R.

Colvin S.C.

Condello S.

Currò $\mathrm{M}$.

D'Adamo S.

Dalla Via L.

De Stefano S.C.

De Feo K.

Del Duca $S$.

Di Pierro P.

Esposito M.

Facchini A.

Facchini A.

Fatima T.

Ferlazzo N.

Filardo G.

Flamigni F.

Frye J.W.

Fratini E.

Galvao F.C.

García-Argáez A.N.

Germani F.

Gilmour S.K.
Giordano E.

Giosafatto C.V.L.

Goyal R.

Grancara S.

Guidotti S.

Handa A.K.

Hayes C.S.

Hermann A.

Higashi K.

Hörkkö S.

Hyvönen M.T.

Ientile R.

Igarashi K.

Iorio $\mathrm{R}$.

Jantunen E.

Johnson L.R.

Juutinen S.

Kaiser A.

Kashiwagi K.

Keinänen T.A.

Khomutov A.R.

Khomutov M.A.

Koistinaho J.

Kurihara S.

Lasanajak Y.

Liu C.

Maier B.

Majumdar R.

Mäkinen P.I.

Manente S.

Mäntymaa $P$.

Mariniello L.

Mariottini P.

Martinis P.

Matsufuji S.

Mattoo A.K.

Merali S

Minguzzi M.

Minocha R.

Minocha S.C.

Mirmira R.G.

Moya-García A.A.

Murai N.

Murakami Y.

Murray-Stewart T.

Obakan P.

Ohkido M.

Palavan-Ünsal N.
Pasini A.

Perez-Leal O.

Pino-Ángeles A.

Pirnes-Karhu S.

Platano D.

Porta R.

Porter C.

Ray R.M.

Risitano R.

Rossi D.

Rossi M.G.

Sakamoto A.

Sánchez-Jiménez F.

Saroj S.D.

Serafini F.D.

Shao L.

Sironen R.

Soleimani M.

Sorrentino A.

Stefanelli C.

Suzuki H.

Takizawa H.

Tempera G.

Tersey S.A.

Terui Y.

Thomas T.J.

Thomas $\mathrm{T}$.

Toida $\mathrm{T}$.

Toninello A.

Trisolino G.

Uimari A.

Urdiales J.L.

Valentini S.R.

Varesio L.

Vecchio M.

Vepsäläinen J.

Verderio E.

Vijayanathan V.

von Koschitzky I.

Wallace H.M.

Weiger T.M.

Wilson K.T.

Wojciechowski S.

Woster P.M.

Yoshida T.

Zahedi K.

Zanelli C.F 


\section{References}

Abdulhussein AA, Wallace HM (2013) Polyamines and membrane transporters. Amino Acids (in this issue). doi:10.1007/s00726013-1553-6

Agostinelli E, Seiler N (2006) Non-irradiation-derived reactive oxygen species (ROS) and cancer: therapeutic implications. Amino Acids 31:341-355

Agostinelli E, Arancia G, Dalla Vedova L, Belli F, Marra M, Salvi M, Toninello A (2004) The biological functions of polyamine oxidation products by amine oxidases perspectives of clinical applications. Amino Acids 27:347-358

Agostinelli E, Belli F, Molinari A, Condello M, Palmigiani P, DallaVedova L, Marra M, Seiler N, Arancia G (2006) Toxicity of enzymatic oxidation products of spermine to human melanoma cells (M14): sensitisation by heat and MDL 72527. Biochim Biophys Acta 1763:1040-1050

Agostinelli E, Condello M, Molinari A, Tempera G, Viceconte N, Arancia G (2009) Cytotoxicity of spermine oxidation products to multidrug resistant melanoma cells (M14 ADR2): sensitisation by MDL 72527 , a lysosomotropic compound. Int $\mathrm{J}$ Oncol 35:485-498

Agostinelli E, Tempera G, Viceconte N, Saccoccio S, Battaglia V, Grancara S, Toninello A, Stevanato R (2010) Potential anticancer application of polyamine oxidation products formed by amine oxidase: a new therapeutic approach. Amino Acids 38:353-368

Amendola R, Cervelli M, Tempera G, Fratini E, Varesio L, Mariottini P, Agostinelli E (2013) Spermine metabolism and radiation derived reactive oxygen species for future therapeutic implications in cancer: an additive or adaptive response. Amino Acids (in this issue). doi:10.1007/s00726-013-1579-9

Arancia G, Calcabrini A, Marra M, Crateri P, Artico M, Martone A, Martelli F, Agostinelli E (2004) Mitochondrial alterations induced by serum amine oxidase and spermine on human multidrug resistant tumor cells. Amino Acids 26:273-282

Atemnkeng VA, Pink M, Schmitz-Spanke S, Wu XJ, Dong LL, Zhao KH, May C, Laufer S, Langer B, Kaiser A (2013) Deoxyhypusine hydroxylase from Plasmodium vivax, the neglected human malaria parasite: molecular cloning expression and specific inhibition.by the 5-LOX inhibitor zileuton. PLoS One 8:58318. doi:10.1371/journal.pone.0058318

Averill-Bates DA, Cherif A, Agostinelli E, Tanel A, Fortier G (2005) Anti-tumoral effect of native and immobilized bovine serum amine oxidase in a mouse melanoma model. Biochem Pharmacol 69:1693-1704

Averill-Bates DA, Ke Q, Tanel A, Roy J, Fortier G, Agostinelli E (2008) Mechanism of cell death induced by spermine and amine oxidase in mouse melanoma cells. Int J Oncol 32:79-88

Bacchi CJ, Yarlett N, Faciane E, Bi X, Rattendi D, Weiss LM, Woster PM (2009) Metabolism and alkyl polyamine analog by a polyamine oxidase from the microsporidian Encephalitozoon Cuniculi. Antimicrob Agents Chemother 53:2599-2604. doi:10. 1128/AAC.00267-08

Battaglia V, De Stefano Shields C, Casero RA Jr (2013) Polyamine catabolism in carcinogenesis: potential targets for chemotherapy and chemoprevention. Amino Acids (in this issue). doi:10.1007/ s00726-013-1529-6

Beninati S, Facchiano F, Piacentini M (2013) Transglutaminases: future perspectives. Amino Acids 44:1-300

Bergamini CM, Collighan RJ, Wang Z, Griffin M (2011) Structure and regulation of type 2 transglutaminase in relation to its physiological functions and pathological roles. Adv Enzymol Relat Areas Mol Biol 78:1-46
Borzì RM, Guidotti S, Minguzzi M, Facchini A, Platano D, Trisolino G, Filardo G, Cetrullo S, D'Adamo S, Stefanelli C, Facchini A, Flamigni F (2013) Polyamine delivery as a tool to modulate stem cell differentiation in skeletal tissue engineering. Amino Acids (in this issue). doi:10.1007/s00726-013-1607-9

Bresciani G, Cruz IB, de Paz JA, Cuevas MJ, González-Gallego J (2013) The MnSOD Ala16Val SNP: relevance to human diseases and interaction with environmental factors. Free Radic Res 47:781-792

Caccamo D, Currò M, Ferlazzo N, Condello S, Ientile R (2012) Monitoring of transglutaminase 2 under different oxidative stress conditions. Amino Acids 42:1037-1043

Calcabrini A, Arancia G, Marra M, Crateri P, Befani O, Martone A, Agostinelli E (2002) Enzymatic oxidation products of spermine induce greater cytotoxic effects on human multidrug-resistant colon carcinoma cells (LoVo) than on their wild type counterparts. Int J Cancer 99:43-52

Casero RA, Woster PM (2009) Recent advances in the development of polyamine analogues as antitumor agents. J Med Chem 52:4551-4573

Castro-Oropeza R, Pino-Ángeles A, Khomutov MA, Urdiales JL, Moya-García AA, Vepsäläinen J, Khomutov A, Sánchez-Jiménez F (2013) Aminooxy analogue of histamine is an efficient inhibitor of mammalian L-histidine decarboxylase: combined in silico and experimental evidences. Amino Acids (in this issue). doi:10.1007/s00726-013-1589-7

Cervelli M, Angelucci E, Germani F, Amendola R, Mariottini P (2013) Inflammation, carcinogenesis and neurodegeneration studies in transgenic animal models for polyamine research. Amino Acids (in this issue). doi:10.1007/s00726-013-1572-3

Chaturvedi R, Asim M, Barry DP, Frye JW, Casero RA Jr, Wilson KT (2013) Spermine oxidase is a regulator of macrophage host response to Helicobacter pylori: enhancement of antimicrobial nitric oxide generation by depletion of spermine. Amino Acids (in this issue). doi:10.1007/s00726-013-1531-z

Chawla S, Kvalnes K, deLong MA, Wickett R, Manga P, Boissy RE (2012) DeoxyArbutin and its derivatives inhibit tyrosinase activity and melanin synthesis without inducing reactive oxygen species or apoptosis. J Drugs Dermatol 11:e28-e34

Chen KY, Canellakis ES (1977) Enzyme regulation in neuroblastoma cells in a salts-glucose medium: induction of ornithine decarboxylase by asparagine and glutamine. Proc Natl Acad Sci USA 74:3791-3795

Currò M, Ferlazzo N, Risitano R, Condello S, Vecchio M, Caccamo D, Ientile R (2013) Transglutaminase 2 and phospholipase A2 interactions in the inflammatory response in human THP-1 monocytes. Amino Acids (in this issue). doi:10.1007/s00726013-1569-y

Del Duca S, Verderio E, Serafini-Fracassini D, Iorio R, Cai G (2013) Extracellular transglutaminase. Amino Acids (in this issue). doi:10.1007/s00726-013-1605-y

Fesus L, Piacentini M (2002) Transglutaminase 2: an enigmatic enzyme with diverse functions. Trends Biochem Sci 27:534-539

Folk JE, Cole PW (1966) Mechanism of action of guinea pig liver transglutaminase. I. Purification and properties of the enzyme: identification of a functional cysteine essential for activity. J Biol Chem 241:5518-5525

Folk JE, Park MH, Chung SI, Schrode J, Lester EP, Cooper HL (1980) Polyamines as physiological substrates for transglutaminases. J Biol Chem 255:3695-3700

Fong LY, Feith DJ, Pegg AE (2003) Antizyme overexpression in transgenic mice reduces cell proliferation, increases apoptosis, and reduces $N$-nitrosomethylbenzylamide-induced forestomach carcinogenesis. Cancer Res 63:3945-3954 
Goldenthal MJ, Marín-García J (2004) Mitochondrial signaling pathways: a receiver/integrator organelle. Mol Cell Biochem 262:1-16

Grancara S, Martinis P, Manente S, García-Argáez AN, Tempera G, Bragadin M, Dalla Via L, Agostinelli E, Toninello A (2013) Bidirectional fluxes of spermine across the mitochondrial membrane. Amino Acids (in this issue). doi:10.1007/s00726013-1591-0

Griffin M, Casadio R, Bergamini CM (2002) Transglutaminases: nature's biological glues. Biochem J 368:377-396

Gundemir S, Colak G, Tucholski J, Johnson GV (2012) Transglutaminase 2: a molecular Swiss army knife. Biochim Biophys Acta 1823:406-419. doi:10.1016/j.bbamcr.2011.09.012

Handa AK, Mattoo AK (2010) Differential and functional interactions emphasize the multiple roles of polyamines in plants. Plant Physiol Biochem 48:540-546

Hauber I, Bevec D, Heukeshoven J, Krätzer F, Horn F, Choidas A, Harrer T, Hauber J (2005) Identification of cellular deoxyhypusine synthase as a novel target for antiretroviral therapy. J Clin Invest 115:76-85

Hayes CS, DeFeo K, Woster PM, Gilmour SK (2013) Elevated ornithine decarboxylase activity promotes skin tumorigenesis by stimulating the recruitment of bulge stem cells but not via toxic polyamine catabolic metabolites. Amino Acids (in this issue). doi:10.1007/s00726-013-1559-0

Keinänen TA, Hyvönen MT, Alhonen L, Vepsäläinen J, Khomutov AR (2013) Selective regulation of polyamine metabolism with methylated polyamine analogues. Amino Acids (in this issue). doi:10.1007/s00726-013-1587-9

Keren-Paz A, Bercovich Z, Porat Z, Erez O, Brener O, Kahana C (2006) Overexpression of antizyme-inhibitor in NIH3T3 fibroblasts provides growth advantage through neutralization of antizyme functions. Oncogene 25:5163-5172

Kim SY, Jeitner TM, Steinert PM (2002) Transglutaminases in disease. Neurochem Int 40:85-103

Kusano T, Berberich T, Tateda C, Takahashi Y (2008) Polyamines: essential factors for growth and survival. Planta 228:367-381

Lasanajak Y, Minocha R, Minocha SC, Goyal R, Fatima T, Handa AK, Mattoo AK (2013) Enhanced flux of substrates into polyamine biosynthesis but not ethylene in tomato fruit engineered with yeast S-adenosylmethionine decarboxylase gene. Amino Acids (in this issue). doi:10.1007/s00726-0131624-8

Lindsay GS, Wallace HM (1999) Changes in polyamine catabolism in HL-60 human promyelogenous leukaemic cells in response to etoposide-induced apoptosis. Biochem J 337:83-87

Liu C, Perez-Leal O, Barrero C, Zahedi K, Soleimani M, Porter C, Merali S (2013) Modulation of polyamine metabolic flux in adipose tissue alters the accumulation of body fat by affecting glucose homeostasis. Amino Acids (in this issue). doi:10.1007/ s00726-013-1548-3

Mangold U (2006) Antizyme inhibitor: mysterious modulator of cell proliferation. Cell Mol Life Sci 63:2095-2101

Mariniello L, Porta R, Sorrentino A, Giosafatto CVL, Rossi Marquez G, Esposito M, Di Pierro P (2013) Transglutaminase-mediated macromolecular assembly: production of conjugates for food and pharmaceutical applications. Amino Acids (in this issue). doi:10. 1007/s00726-013-1561-6

Marton LJ, Pegg AE (1995) Polyamines as targets for therapeutic intervention. Annu Rev Pharmacol Toxicol 35:55-91

Minarini A, Milelli A, Tumiatti V, Rosini M, Bolognesi ML, Melchiorre C (2010) Synthetic polyamines: an overview of their multiple biological activities. Amino Acids 38:383-392

Murakami Y, Ohkido M, Takizawa H, Murai N, Matsufuji S (2013) Multiple forms of mouse antizyme inhibitor 1 mRNA differentially regulated by polyamines. Amino Acids (in this issue). doi:10.1007/s00726-013-1598-6

Murray-Stewart T, Woster PM, Casero RA Jr (2013) The reexpression of the epigenetically silenced $e$-cadherin gene by a polyamine analogue lysine-specific demethylase-1 (LSD1) inhibitor in human acute myeloid leukemia cell lines. Amino Acids (in this issue). doi:10.1007/s00726-013-1485-1

Nishiki Y, Farb TB, Friedrich J, Bokvist K, Mirmira RG, Maier B (2013) Characterization of a novel polyclonal polanti-hypusine antibody. Springerplus 2:421. doi:10.1186/2193-1801-2-421

Nishimura Y, Ishii J, Okazaki F, Ogino C, Kondo A (2012) Complex carriers of affibody-displaying bio-nanocapsules and composition-varied liposomes for HER2-expressing breast cancer cellspecific protein delivery. J Drug Targ 20:897-905

Obakan P, Arısan ED, Calcabrini A, Agostinelli E, Bolkent Ş, PalavanÜnsal N (2013) Activation of polyamine catabolic enzymes involved in diverse responses against epibrassinolide-induced apoptosis in LNCaP and DU145 prostate cancer cell lines. Amino Acids (in this issue). doi:10.1007/s00726-013-1574-1

Park D, Choi SS, Ha KS (2010) Transglutaminase 2: a multifunctional protein in multiple subcellular compartments. Amino Acids 39:619-631

Pasini A, Caldarera CM, Giordano E (2013) Chromatin remodelling by polyamines and polyamine analogues. Amino Acids (in this issue). doi:10.1007/s00726-013-1550-9

Pignatti C, Tantini B, Stefanelli C, Flamigni F (2004) Signal transduction pathways linking polyamines to apoptosis. Amino Acids 27:359-365

Pirnes-Karhu S, Mäntymaa P, Sironen R, Mäkinen PI, Wojciechowski S, Juutinen S, Koistinaho J, Hörkkö S, Jantunen E, Alhonen L, Uimari A (2013) Enhanced polyamine catabolism disturbs hematopoietic lineage commitment and leads to a myeloproliferative disease in mice overexpressing spermidine/spermine $N^{1}$ acetyltransferase. Amino Acids (in this issue). doi:10.1007/ s00726-013-1546-5

Preeti Tapas S, Kumar P, Madhubala R, Tomar S (2013) Structural insight into DFMO resistant ornithine decarboxylase from Entamoeba histolytica: an inkling to adaptive evolution. PLoS One 8:53397. doi:10.1371/journal.pone.0053397

Raj KP, Zell JA, Rock CL, McLaren CE, Zoumas-Morse C, Gerner EW, Meyskens FL (2013) Role of dietary polyamines in a phase III clinical trial of difluoromethylornithine (DFMO) and sulindac for prevention of sporadic colorectal adenomas. $\mathrm{Br} \mathrm{J}$ Cancer 108:512-518

Ray RM, Johnson LR (2013) Regulation of intestinal mucosal growth by amino acids. Amino Acids (in this issue). doi:10.1007/ s00726-013-1565-2

Ray RM, Viar MJ, Patel TB, Johnson LR (1999) Interaction of asparagine and EGF in the regulation of ornithine decarboxylase in IEC-6 cells. Am J Physiol 276:G773-G780

Ray RM, Viar MJ, Johnson LR (2012) Amino acids regulate the expression of antizyme-1 to modulate ornithine decarboxylase activity. J Biol Chem 287:3674-3690

Rossi D, Carrilho Galvão F, Bellato HM, Boldrin P, Andrews BJ, Valentini SR, Zanelli CF (2013) eIF5A has a function in the cotranslational translocation of proteins into the ER. Amino Acids (in this issue). doi:10.1007/s00726-013-1618-6

Sarkar NK, Clarke DD, Waelsch H (1957) An enzymically catalyzed incorporation of amines into proteins. Biochim Biophys Acta 25:451-452

Schwentke A, Krepstakies M, Mueller AK, Hammerschmidt-Kamper C, Motaal BA, Bernhard T, Hauber J, Kaiser A (2012) In vitro and in vivo silencing of plasmodial dhs and eIF-5a genes in a putative, non-canonical RNAi-related pathway. BMC Microbiol 13:12-107 
Seiler N (1992) Polyamine catabolism and elimination by the vertebrate organism. In: Dowling RH, Fölsch UR, Löser G (eds) Polyamines in the gastrointestinal tract Kluwer Academic Publishers. Boston, London, Dordrecht, pp 65-85

Seiler N, Raul F (2005) Polyamines and apoptosis. J Cell Mol Med 9:623-642

Shao L, Bhatnagar P, Majumdar R, Minocha R, Minocha SC (2013) Putrescine overproduction does not affect the catabolism of spermidine and spermine in poplar and Arabidopsis. Amino Acids (in this issue). doi:10.1007/s00726-013-1581-2

Sinigaglia G, Magro M, Miotto G, Cardillo S, Agostinelli E, Zboril R, Bidollari E, Vianello F (2012) Catalytically active bovine serum amine oxidase bound to fluorescent and magnetically drivable nanoparticles. Int $\mathbf{J}$ of Nanomed 7:1-11

Szondy Z, Korponay-Szabó I, Király R, Fésüs L (2011) Transglutaminase 2 dysfunctions in the development of autoimmune disorders: celiac disease and TG2-/- mouse. Adv Enzymol Relat Areas Mol Biol 78:295-345

Telci D, Griffin M (2006) Tissue transglutaminase (TG2)-a wound response enzyme. Front Biosci 11:867-882

Tersey SA, Colvin SC, Maier B, Mirmira RG (2013) Protective effects of polyamine depletion in mouse models of type 1 diabetes: implications for therapy. Amino Acids (in this issue). doi:10.1007/s00726-013-1560-7

Terui Y, Saroj SD, Sakamoto A, Yoshida T, Higashi K, Kurihara S, Suzuki H, Toida T, Kashiwagi K, Igarashi K (2013) Properties of putrescine uptake by PotFGHI and PuuP and their physiological significance in Escherichia coli. Amino Acids (in this issue). doi:10.1007/s00726-013-1517-x

Thomas T, Thomas TJ (2001) Polyamines in cell growth and cell death: molecular mechanisms and therapeutic applications. Cell Mol Life Sci 58:244-258
Thomas RM, Thomas T, Wada M, Sigal LH, Shirahata A, Thomas TJ (1999) Facilitation of the cellular uptake of a triplex-forming oligonucleotide by novel polyamine analogues: structure-activity relationships. Biochemistry 38:13328-13337

Tobias KE, Kahana C (1995) Exposure to ornithine results in excessive accumulation of putrescine and apoptotic cell death in ornithine decarboxylase overproducing in mouse myeloma cells. Cell Growth Differ 6(1279):1285

Toninello A, Salvi M, Mondovì B (2004) Interaction of biologically active amines with mitochondria and their role in the mitochondrial-mediated pathway of apoptosis. Curr Med Chem 11:2349-2374

Vijayanathan V, Thomas T, Thomas TJ (2002) DNA nanoparticles and development of DNA delivery vehicles for gene therapy. Biochemistry 41:14085-14094

Vijayanathan V, Agostinelli E, Thomas T, Thomas TJ (2013) Innovative approaches to the use of polyamines for DNA nanoparticles preparation for gene therapy. Amino Acids (in this issue). doi:10.1007/s00726-013-1549-2

Von Koschitzky I, Kaiser A (2013) Chemical profiling of deoxyhypusine hydroxilase inhibitors for antimalarian therapy. Amino Acids 45:1047-1053

Weiger TM, Hermann A (2013) Cell proliferation, potassium channels, polyamines and their interactions, a mini review. Amino Acids (in this issue). doi:10.1007/s00726-013-1536-7

Zhu Q, Huang Y, Marton LJ, Woster PM, Davidson NE, Casero RA Jr (2012) Polyamine analogues modulate gene expression by inhibiting Lysine-Specific Demethylase 1 (LSD1) and altering chromatin structure in human breast cancer cells. Amino Acids 42:887-898. doi:10.1007/s00726-011-1004-1 